\title{
Addressing the challenges of refugee health: Experiences from the University of Louisville interprofessional refugee immunization clinic
}

\author{
Ruth Carrico ${ }^{* 1}$, Rahel Bosson ${ }^{1}$, Jennifer Koch ${ }^{2}$, Anupama Raghuram ${ }^{1}$, Paula Peyrani ${ }^{1}$, Rebecca Ford ${ }^{1}$, Allison Pauly ${ }^{3}$, \\ Katherine Contreras Rivera ${ }^{1}$, Taghreed Abdulmogith ${ }^{1}$, William Johnson ${ }^{1}$, Sarah C. Van Heiden ${ }^{1}$, Dawn Balcom ${ }^{1}$, \\ Montray Smith ${ }^{4}$, Timothy Wiemken ${ }^{1}$, Julio Ramirez ${ }^{1}$ \\ ${ }^{1}$ Global Health Center, Division of Infectious Diseases, School of Medicine, University of Louisville, Louisville, KY, United States \\ ${ }^{2}$ Division of Internal Medicine, School of Medicine, University of Louisville, United States \\ ${ }^{3}$ Kentucky Office for Refugees, Louisville, KY, United States \\ ${ }^{4}$ School of Nursing, University of Louisville, United States
}

Received: June 1, 2015

DOI: $10.5430 /$ jnep.v5n12p88
Accepted: August 30, $2015 \quad$ Online Published: September 22, 2015

URL: http://dx.doi.org/10.5430/jnep.v5n12p88

\begin{abstract}
Addressing the health needs among refugee populations requires an approach that recognizes social, cultural and language issues and has a platform for interventions that actively use the strengths and competencies from a variety of healthcare disciplines. An interprofessional practice model was implemented in 2012 at the University of Louisville as part of a program aimed at providing age-appropriate vaccines for newly arriving refugees in the Louisville community. During 2012-2014, the program involved more than 35 faculty, 22 residents and fellows, 278 students, 28 support staff and 16 researchers in collaborative work representing nursing, medicine, public health, pharmacy, and social services disciplines in addition to students and faculty from business and engineering. As of early 2015, more than 20,000 doses of vaccine have been safely provided to more than 5,000 refugees. The processes and outcomes have been deemed as beneficial by the refugees and healthcare providers. This type of interprofessional practice model may serve as a deliberative learning platform for other refugee health services.
\end{abstract}

Key Words: Refugee immunization, Vaccination, Health, Interprofessional practice, Interprofessional education

\section{INTRODUCTION}

According to the Department of Homeland Security, approximately 75,000 refugees and 450,000 legal permanent immigrants are resettled into the United States each year and represent nearly every country in the world and a majority of spoken languages and cultures. ${ }^{[1,2]}$ According to the Catholic Charities of Louisville, Inc. (CC) and Kentucky Office for Refugees (KOR), during the calendar year 2013, almost 2,500 refugees arrived in Kentucky as part of the fed- eral refugee resettlement program. Within this group, over $40 \%$ arrived from Cuba, $12 \%$ from Iraq, $11 \%$ from Bhutan, $8 \%$ from Somalia, $8 \%$ from Burma/Myanmar. In addition, refugees entered in smaller numbers from the Democratic Republic of the Congo, Ethiopia, Afghanistan, Sudan, Rwanda, Burundi, Djibouti, Kenya, and Syria. These groups represent a variety of cultures and an array of languages are spoken including; Spanish, Arabic, Nepali, Somali, Swahili, Karen, Burmese, Chin, Amharic, Oromo, Russian and Kinyarwanda.

${ }^{*}$ Correspondence: Ruth Carrico; Email: ruth.carrico@louisville.edu; Address: Global Health Center, Division of Infectious Diseases, School of Medicine, University of Louisville, Louisville, KY, United States 
Refugees arriving in Kentucky were placed in one of four cities with 1,800 (72\%) being placed in Louisville and the remainder in Bowling Green, Lexington, or Owensboro (Kentucky Office of Refugees, personal communication, 2014). The overarching problem, as well as the opportunity, involves how to address the diversity of health issues within the challenging social and economic framework posed by broad language differences and cultural backgrounds. Further, accomplishing this through a practice and educational process that is sustainable and consistent requires a new perspective for the education and training of all healthcare personnel participating in the resettlement process. Little is written about interprofessional approaches in care of refugees, but the need for approaches that capture a full range of skills has been recognized. The importance of a workforce that can address language barriers, differences in living skills, and the expectations of the individual refugee groups was described by Australian researchers. ${ }^{[3]}$ The provision of dental care for refugees using teams of dentists, physicians, and nurses resulted in a holistic approach for care that improved access to dental care for the refugees while increasing humanitarian values among the healthcare participants. ${ }^{[4]}$ Oregon Health \& Science University implemented interdisciplinary elective courses focused on activities provided within refugee community centers. ${ }^{[5]}$ Each of these authors recognized the importance of bringing multiple disciplines together to provide a more expansive service approach while enabling a richer educational experience for the student participants. Improved access to care and service as well as an emphasis on disease prevention and health promotion were also common threads across those three reports.

With healthcare reform, the ability of individuals to access care is changing and the emphasis on prevention and health promotion has taken on new significance. The ability of the healthcare infrastructure to address unique health needs within a construct that acknowledges and addresses language, culture, social and economic barriers and differences will be critical in the provision of quality and affordable care. Germaine to this issue are new practice and education techniques that actively enable the healthcare workforce to provide care that is culturally competent, inclusive, and of acceptable and consistent quality. Accomplishing this necessitates a methodology that is actively collaborative and in recognition of the varied skill sets and competencies of all participants.

In 2012, clinicians and researchers at the University of Louisville entered into a partnership with $\mathrm{CC}$ and KOR aimed at providing real-time assessments of the health conditions present among refugees resettling in Kentucky. The first phase of this partnership involved development of reports that summarized the results of initial health screenings

Published by Sciedu Press provided to the newly arriving refugees. It was quickly determined that issues relevant to the care and safety of the refugees as well as the public health of the communities welcoming those refugees could be improved through a new approach to the provision of care, assessment of illness and health need, and prevention through immunization. Each incoming refugee, both adult and child, needed to receive age-appropriate vaccines, not only for public health reasons but also to fulfill the requirement by the United States Citizenship and Immigration Services (USCIS) that all refugees receive these vaccines before becoming eligible to apply for a permanent residency card (Green Card) and eventual US citizenship. ${ }^{[6]}$ Limited information regarding immunization history was available and came primarily from overseas medical examinations provided in the refugees' country of origin. Although a medical examination was performed, its focus was on identification of illnesses that actively represented a risk to the US population, but not on susceptibility to vaccine-preventable diseases. That responsibility is borne by the receiving agencies involved in resettlement. The challenge for the local community in Louisville, Kentucky included determination of needed vaccines, recognition of individual health conditions, development of a method of care that addressed cultural and language differences, provision of immunization in a safe manner, and performance of these activities in a way that recognized limited financial and workforce resources. The opportunity involved conceptualization then implementation of a novel approach that brought together relevant health professionals to form the Interprofessional Refugee Immunization Clinic. The clinic supported a method for provision of high quality immunization care to the refugees while providing a deliberative learning environment based upon interprofessional education and practice. The objective of this manuscript is to describe the development, implementation, and outcomes of the University of Louisville Interprofessional Refugee Immunization Clinic.

\section{METHOD}

\subsection{Interprofessional refugee immunization clinic: De- velopment}

The initial reporting summarizing health conditions among newly arriving refugees identified the inability to determine their immunization rates. A pilot program was proposed that included performance and financial metrics. The performance goal was to develop an immunization process that would enable access by all refugees during their eight month resettlement period. The financial goal was accomplishment of immunization without exceeding funds available for those purposes. Upon acceptance of this program, in late 2012/early 2013, the University of Louisville School of 
Medicine undertook the planning, development and implementation of a refugee immunization program that was based upon a model of interprofessional practice. The objectives of the program included: 1) development of an immunization process that addressed the age-appropriate vaccines outlined in the Centers for Disease Control and Prevention (CDC) Global Migration and Quarantine guidelines and Health and Human Services Office of Refugee Resettlement guidelines1; and 2) alignment of the process with the interprofessional education and collaboration goals outlined by the $2011 \mathrm{ex}-$ pert panel report that followed recommendations from the Institute of Medicine. ${ }^{[7,8]}$

Using input from students from the UL Speed School of Engineering, workflow processes were designed and simulations conducted to identify aspects that were prone to error or safety risks and delays in throughput. Public Health students helped identify steps in the process that posed challenges for addressing the cultural needs of various refugee groups, for example privacy needs of Muslim women during vaccination, and health literacy needs for refugees who are illiterate. Students in medicine, nursing, and pharmacy helped develop the initial immunization procedures recognizing the constraints posed by the physical environment including small work spaces, lack of immediate access to sinks for hand hygiene, environmental temperature variations, and space for emergency response if needed. Roleplaying provided opportunities for student groups to work together, ask clarifying questions, demonstrate competence in their roles and responsibilities, and experience the full process prior to implementation.

\subsection{Interprofessional refugee immunization clinic: Im- plementation}

\subsubsection{The immunization process}

An overarching goal for the clinic was safe provision and documentation of all age-appropriate vaccines recommended by CDC for all refugees resettling in the Louisville area. For most adults, these vaccines included: 1 ) the 3 -dose primary tetanus series including two doses of $\mathrm{Td}$ and one dose of tdap; 2) two doses of the measles-mumps-rubella (MMR) vaccine; 3) two doses of varicella vaccine unless there was a documented positive titer; and 4) one dose of influenza vaccine during the influenza season. One dose of pneumococcal vaccine might also be administered depending upon patient's age and underlying health conditions. Ten months into the clinic program, provision of vaccines for the pediatric and adolescent population was added to the program due to the need to ensure immunization prior to school entry. These vaccines included all routine childhood immunizations according to current Advisory Committee on Immunization
Practices (ACIP) recommendations. Vaccines for adults, adolescents and children were selected based upon reliable documentation of prior doses and health indicators such as titer results, age, underlying medical conditions, and contraindications. Accomplishing this task required that all participating healthcare personnel achieve the following competencies: 1) knowledge of the vaccines and the disease they prevent; 2) ability to select the correct vaccine(s) for each individual based upon the ACIP recommendations including dose and site of administration; 3) ability to handle the vaccine while maintaining cold chain; 4) use of safe injection practices during mixing, drawing the administration dose; administration of the vaccine; use of safety devices; and disposal of equipment; 5) querying and educating the patient recipient or parent of the vaccine recipient via the use of onsite interpreters or a telephone language line; 6) culturally sensitive administration techniques such as offering privacy for Muslim women and distraction techniques for children; 7) monitoring of the vaccine recipient post-immunization; and 8) documentation.

Follow up post-immunization was an important part of the process in that adverse events may occur or health conditions may change necessitating an alteration in future immunization plans. Recognizing this, improvements in the system that involved development of individual immunization plans emerged in order to facilitate a nimble approach for each refugee. Some methods of documenting identifying information, health information obtained during decision-making prior to immunization, existing titer results, and vaccine administration needed to be developed. A vaccine "trip tik" was subsequently crafted (see Figure 1) so health information relevant to immunization, prior receipt of vaccines, and documentation of administered vaccines could follow the individual refugee patient through the process and be maintained in a database for retrieval and ongoing quality monitoring. The vaccine "trip tik" formed a basis for assessment, documentation, translation, education, training, and health information sharing. Nursing, medicine, pharmacy, and public health faculty collaborated in development of this "trip tik" and in its subsequent improvements.

\subsubsection{Alignment of the process with interprofessional edu- cation and collaboration goals}

Collaboration among the health professions is not a new concept. Since the early 1970's, there has been an emphasis on interdisciplinary approaches to the teaching of health sciences students. The desire to link education with the practical elements of care, and develop new faculty skills that enable them to use cooperative models during the education process has been a continuing theme, particularly within academic health centers. This was voiced specifically 
in the 1972 Institute of Medicine report "Educating for the Health Team", ${ }^{[3]}$ and more recently in a 2011 expert panel report "Core Competencies for Interprofessional Collaborative Practice". ${ }^{[4]}$ The need for opportunities where a variety of health disciplines can come together to address complex problems in an environment that supports deliberative work- ing and learning has represented educational and practice nirvana. This clinic offered a unique opportunity to bring health and other related disciplines together to work on common goals - the provision of high quality healthcare for a vulnerable, yet resilient, international population.
DOA: Exp:

Last Name:

A \#:
Green Card Vaccines "Trip-Tik"

First Name:

\section{Language:}

\begin{tabular}{|c|c|c|}
\hline Questions to Ask & Yes & No \\
\hline Are you sick today? & & \\
\hline Do you have allergies to medication, or certain foods? & & \\
\hline Have you ever had a serious reaction after you received a vaccine? & & \\
\hline $\begin{array}{l}\text { Do you have heart problems, lung problems, asthma, kidney problems, diabetes, anemia or another } \\
\text { long term sickness? }\end{array}$ & & \\
\hline Do you have cancer, or any other problem with your body's defense (immune system)? & & \\
\hline $\begin{array}{l}\text { In the past } 3 \text { months have you taken medicine that weakens your body's defense (immune system)? } \\
\text { E.g. steroids - cortisone, prednisone, medicine to fight cancer or radiation treatment }\end{array}$ & & \\
\hline Have you ever had a seizure (fits) or any problem with your brain or nervous system? & & \\
\hline $\begin{array}{l}\text { In the past year have you received a blood transfusion, been given immune globulin or an antiviral } \\
\text { drug (medicine to fight a virus)? }\end{array}$ & & \\
\hline Are you pregnant? Any chance you could be pregnant? & & \\
\hline Do you have any vaccine records with you? & & \\
\hline
\end{tabular}

Vaccines you need today:

\begin{tabular}{|c|c|c|c|c|c|c|}
\hline Vaccine & Dosage & Date & Brand & $\begin{array}{c}\text { Lot } \\
\text { Number }\end{array}$ & Site & Administered by \\
\hline Influenza & Dose 1 & & & & & \\
\hline Tdap & Dose 1 & & & & & \\
\hline $\mathrm{Td}$ & Dose 1 & & & & & \\
\hline \multirow{2}{*}{ MMR } & $\square$ Dose 1 & & & & & \\
\hline & $\square$ Dose 2 & & & & & \\
\hline \multirow{2}{*}{ Varicella } & $\square$ Dose 1 & & & & & \\
\hline & $\square$ Dose 2 & & & & & \\
\hline \multirow{3}{*}{ Hepatitis B } & $\square$ Dose 1 & & & & & \\
\hline & $\square$ Dose 2 & & & & & \\
\hline & $\square$ Dose 3 & & & & & \\
\hline
\end{tabular}

Provider:

Date:

Your next appointment for more immunizations is scheduled for:

Kentucky Office for Refugees

University of Louisville Vaccine and International Travel Center

502-562-2822

Rev: 6/2014

Figure 1. Green Card Vaccines "Trip-Tik" 
The interprofessional education and collaboration goals mirrored those outlined by the 2011 expert panel report in terms of desired principles that underpin competencies including care that is: 1) Patient/family centered; 2) Community/population oriented; 3) Relationship focused; 4) Process oriented; 5) Linked to learning activities educational strategies, and behavioral assessments that are developmentally appropriate for the learner; 6) Able to be integrated across the learning continuum; 7) Sensitive to the systems context/applicable across practice settings; 8) Applicable across professions; 9) Stated in language common and meaningful across the professions; and 10) Outcome driven. ${ }^{[8]}$

Each of the participating disciplines had specific areas of responsibilities with the clinic. Public health professionals were responsible for addressing the cultural needs of each refugee including arranging logistics for interpreters, vaccine information statements in each language with verbal review for those unable to read their native language, and movement through the clinic from check-in to check-out. Nursing was responsible for administration of each dose of vaccine, educating each vaccine recipient, and documentation of each dose received. Pharmacists were responsible for monitoring the environmental conditions of the vaccine, distribution of vaccine doses, and addressing questions regarding drug-vaccine interactions. Medicine was responsible for adherence with the vaccine schedules, addressing questions concerning contraindications, and stand-by for any emergency response necessary in the event of syncopal episodes or vaccine reaction. Engineering students helped develop the process through simulation and implemented adjustments based upon recognized "choke points" in the process flow. Roleplaying and simulation before the first vaccine clinic as well as post-event hot wash discussions helped identify areas of concern as well as areas of high performance and helped bring a sense of high fidelity to the process.

The immunization clinics were situated in the community centers that are the hub of activities for the resettled refugee. This enabled parents and children to come for immunization together and at times convenient for them with work and school constraints. Scheduling of the clinics focused on enabling refugees to attend with others from their home country. This facilitated the ability to address specific cultural and language needs among each population and incorporate strategies to build trusted relationships. Use of expertise from the business and engineering students and faculty helped to ensure processes that were efficient and safe while also addressing consistent and appropriate use of limited human and material resources. Activities specific to the immunization process were linked with measurable competencies and the provision of immunization afforded the opportunity for the various disciplines to work together and learn from each other. Figure 2 provides an example of a skills validation checklist used in the multidisciplinary competence assessment. Use of the checklist enabled opportunities for real-time intervention and practice redirection.

\section{RESUlts}

The immunization program used in the clinic was designed to be a real-time interprofessional practice environment that began with representation from nursing, medicine, pharmacy and public health but expanded over the course of twelve months to include students and faculty from infectious diseases, pediatrics, internal medicine and social services totaling more than 35 different faculty, 22 residents and fellows, 278 students, 28 support staff and 16 researchers. A survey was provided to the initial 200 students and 30 faculty in an effort to gather perceived benefits from the process and enable a $100 \%$ survey response rate. Among those 230 respondents, $96 \%(221 / 230)$ indicated their satisfaction with the process; $100 \%$ indicated that they obtained new knowledge; $100 \%$ perceived their knowledge and application of cultural awareness and sensitivity improved; 94\% (216/230) indicated they learned something new about the other participating disciplines; $100 \%$ indicated their skill set and competence regarding vaccine handling and administration improved; and 96\% (221/230) indicated they found professional value in the opportunity. Interestingly, $100 \%$ of the responding faculty indicated that they learned something new about the other disciplines during their participation in the immunization clinic.

During the first twelve months of the project, more than 5,000 doses of vaccine were provided to more than 1,200 adult refugees. Two episodes of administration errors and six episodes of fainting among the vaccine recipients were identified. No needle stick or other injuries were reported by the participating health discipline students, residents, fellows or faculty. In the first two months of the project where more than 160 pediatric refugees were immunized, more than 600 doses of vaccine were administered. No errors or adverse events were reported among those children. On-site interpreters, language line connections, and health professionals from several of the countries represented by the refugee population helped facilitate adequate and effective communication among the refugees and the health professionals. Communication with case workers helped to ensure that there was a process to report adverse events or concerns with the process among the refugee participants. As of May 2015, more than 20,000 doses of vaccine have been provided to more than 5,000 adult and pediatric refugees using this interprofessional process. 


\section{Skills Validation For}

Intramuscular Immunization Administration (adult)

\begin{tabular}{|c|c|c|}
\hline $\begin{array}{l}\text { Measurement Criteria } \\
\end{array}$ & Met & Not Met \\
\hline 1. Verifies correct medication & & \\
\hline 2. Identifies recipient and assesses for contraindications & & \\
\hline $\begin{array}{l}\text { 3. Ensures vaccine cold chain assessing appropriate vaccine environment (refrigerated } \\
\text { vs. frozen) }\end{array}$ & & \\
\hline 4. Verifies correct dosage according to vaccine used and age of the recipient & & \\
\hline $\begin{array}{l}\text { 5. Selects } 22-23 \text { gauge } \mathrm{x} \text { 1or } 1-1 / 2 \text { inch as standard to facilitate an intramuscular } \\
\text { injection }\end{array}$ & & \\
\hline $\begin{array}{l}\text { 6. Able to attached appropriately sized needle to prefilled syringe without } \\
\text { contamination }\end{array}$ & & \\
\hline $\begin{array}{l}\text { 7. Able to draw correct amount of vaccine into syringe using appropriate technique } \\
\text { (handling syringe, connecting needle, disinfecting top of vial, withdrawing vaccine, } \\
\text { recapping clean needle) if not using prefilled syringe. }\end{array}$ & & \\
\hline 8. Able to correctly describe activation of safety device to prevent needlestick & & \\
\hline 9. Sanitizes hands (alcohol-based hand rub or hand wash) & & \\
\hline $\begin{array}{l}\text { 10. Identifies deltoid muscle and is able to identify the triangular muscle region } \\
\text { appropriate for use in the injection }\end{array}$ & & \\
\hline $\begin{array}{l}\text { 11. Insures that there is no constricting clothing around upper arm (or thigh if } \\
\text { appropriate) area }\end{array}$ & & \\
\hline 12. Helps patient relax arm (or altemate area such as thigh) to decrease muscle tension & & \\
\hline 13. Cleanses injection area with sterile alcohol swab & & \\
\hline $\begin{array}{l}\text { 14. Uses "press and spread" method to insure an intramuscular injection in the deltoid } \\
\text { or thigh muscle }\end{array}$ & & \\
\hline $\begin{array}{l}\text { 15. Inserts needle at } 90^{\circ} \text { angle and, while maintaining control over needle, aspirates to } \\
\text { ensure needle tip is not in a vein }\end{array}$ & & \\
\hline 16. Injects vaccine ensure that entire dose is administered. & & \\
\hline $\begin{array}{l}\text { 17. Activates safety device immediately upon removal of needle from injection site } \\
\text { and disposes of device and syringe in designated sharps container }\end{array}$ & & \\
\hline 18. After injection, gently massages area and applies latex safe band-aid & & \\
\hline $\begin{array}{l}\text { Instructs patient or family/caregiver to move arm and periodically massage } \\
\text { injection area during the next } 24-48 \text { hours to minimize soreness }\end{array}$ & & \\
\hline $\begin{array}{l}\text { 20. Provides patient/family/caregiver education information regarding influenza } \\
\text { vaccine }\end{array}$ & & \\
\hline $\begin{array}{l}\text { 21. Provides patient/family/caregiver with information regarding followup vaccine } \\
\text { doses, as indicated }\end{array}$ & & \\
\hline $\begin{array}{l}\text { 22. Maintains personal protective equipment and emergency pharmaceutical agents } \\
\text { within easy reach }\end{array}$ & & \\
\hline 23. Able to verbally demonstrate appropriate use of emergency protocols & & \\
\hline 24. Able to verbalize documentation required for vaccine administration & & \\
\hline
\end{tabular}

Validation for:

Validation verified by:
Date

Date

(Rev 0715)

Figure 2. Managers' and nurses' competence assessments

Challenges identified included the variation in existing knowledge and skill regarding vaccine administration among the participating schools of nursing, existing health literacy among the refugees concerning health conditions that may represent vaccine contraindication, and the different process flow implemented in the vaccine clinics held at two different sites. To assist schools of nursing with pre-clinic education and skill validation for their students, demonstration 
videos and competency checklists were developed and made available to faculty and students. Addressing health literacy issues continues to be a challenge but one step taken to address it involved the hiring of former refugees to participate in the vaccine clinics as "connectors". Their presence provided assistance to refugee families as they transitioned into healthcare in their new community.

\section{Discussion}

The Interprofessional Refugee Immunization Clinic evolved to include both adults and pediatric refugees in an effort to meet medical and social needs of this population. Further, barriers to successful integration into the community could be addressed as a family. One important example involved the ability to ensure that children received vaccines necessary to attend school thereby enabling parents to go to work and continue their path toward self-sufficiency. From an interprofessional education perspective, this enabled active learning among adult and pediatric practitioners, pharmacists, and public health professionals. The processes in the refugee immunization clinic enabled active learning among adult and pediatric healthcare professionals, pharmacists and public health professionals. This served to underscore the importance of active participation in issues such as vaccinepreventable diseases and the importance of immunization. The health professionals worked together to consider approaches to immunization guided by individual needs while ensuring public health protection. Real-time circumstances and active clinical environments provided situations where these health professionals could problem-solve, experience the competencies and role expectations of the varied disciplines, and practice professional interactions. The clinic also demonstrated process development and the importance of work practice standards and continuous evaluation. A number of process improvements came directly from the health profession students as they discovered new ways of approaching problems. Their ability to recognize best practices and deviations, develop rapid interventions, and assess impact were achievements voiced by participants. Each student and faculty participant observed and experienced the language and cultural realities involved in refugee healthcare. Although each refugee will need to enter American society, they will do so within their existing cultural being. It is critical that each participating healthcare student and faculty recognize the importance of cultural competencies and actively learn how to ensure it as this is a cornerstone to their individual practice. In the provision of immunization this is evident in situations such as administration of vaccine to a
Muslim woman. Ensuring that she has the privacy necessary to enable the healthcare worker to visualize the injection site, provide coverage with her clothing, and have another woman administering the vaccine was an important demonstration of a common cultural difference requiring a culturally sensitive approach. It was important for all clinic participants to recognize this need and develop their own approaches to find the common grounds necessary for trust and ongoing relationships. A number of interesting projects emerged from the collaborations including development of multidisciplinary health promotion projects, poster presentations, and multidisciplinary study groups. Further, the School of Medicine has expanded their existing global health distinction pathway as well as a rotation focused on social justice with both including participation in the refugee immunization clinic.

\section{Conclusion}

In order to address the needs of patients in a changing community, the competencies of its healthcare and public health workforce must also change. Not only must training and education recognize cultural and language diversity, but in order to adequately address those needs, the healthcare and public health workforce must refine their abilities to work together. Through an embracing of interprofessional education and practice, necessary steps can be taken toward this goal. Use of real-time learning environments, such as those provided by our novel Interprofessional Refugee Immunization Clinic, enables this type of practice and learning to occur locally. Helping the refugees address health-related issues and move toward a sustainable and healthy lifestyle represents an invaluable and transformative opportunity for them and for the professionals involved in their care.

\section{ACKNOWLEDGEMENTS}

The authors wish to express thanks to faculty, staff and students from the University of Louisville, Bellarmine University, Spalding University, and Galen Schools of Nursing; the University of Louisville School of Medicine; the University of Louisville College of Business and Speed School of Engineering; the University of Louisville Kent School of Social Work; Sullivan College of Pharmacy; the Kentucky Office for Refugees; Catholic Charities; Kentucky Refugee Ministries; and Migration and Refugee Services for the depth of their collaboration and willingness to explore new approaches with the full spectrum of healthcare disciplines.

\section{CONFLicts OF InTEREST Disclosure}

The authors declare that there is no conflict of interest. 


\section{REFERENCES}

[1] Centers for Disease Control and Prevention Division of Global Migration and Quarantine. Guidelines for the U.S. Domestic Medical Examination for Newly Arriving Refugees. 2012. Available from: http://www.cdc.gov/immigrantrefugeehealth/gui delines/domestic/domestic-guidelines.html

[2] US Department of Homeland Security. Yearbook of immigration statistics. Washington, DC: US Department of Homeland Security; 2010. Available from: http://www.dhs.gov/publication/yearbook-immigra tion-statistics-2013-refugees-and-asylees

[3] Duncan GM, Shepherd M, Symons J. Working with refugees-a manual for caseworkers and volunteers. Rural Remote Health. 2010; 10(4): 1406. PMid:21121699

[4] Farokhi MR, Glass BJ, Gureckis KM. A student operated, faculty mentored dental clinic service experience at the University of Texas Health Science Center at San Antonio for the underserved refugee community: an interprofessional approach. Tex Dent J. 2014; 131(1): 27-33. PMid:24654394

[5] Palmer VS, Mazumder R, Spencer PS. Interprofessional global health education in a cosmopolitan community of North America: the iCHEE experience. Acad Med. 2014; 89(8): 1149 52. PMid:24918760 http://dx.doi.org/10.1097/ACM.00000 00000000363

[6] Immigration and Nationality Act (INA), section. 2012. Available from: http://www.uscis.gov/iframe/ilink/docView/SLB /HTML/SLB/act.html

[7] Institute of Medicine. Educating for the heath team. Washington, DC: National Academy of Sciences; 1972.

[8] Interprofessional Education Collaborative Expert Panel. Core competencies for interprofessional collaborative practice: Report of an expert panel. Washington, DC: Interprofessional Education Collaborative. 2011. 